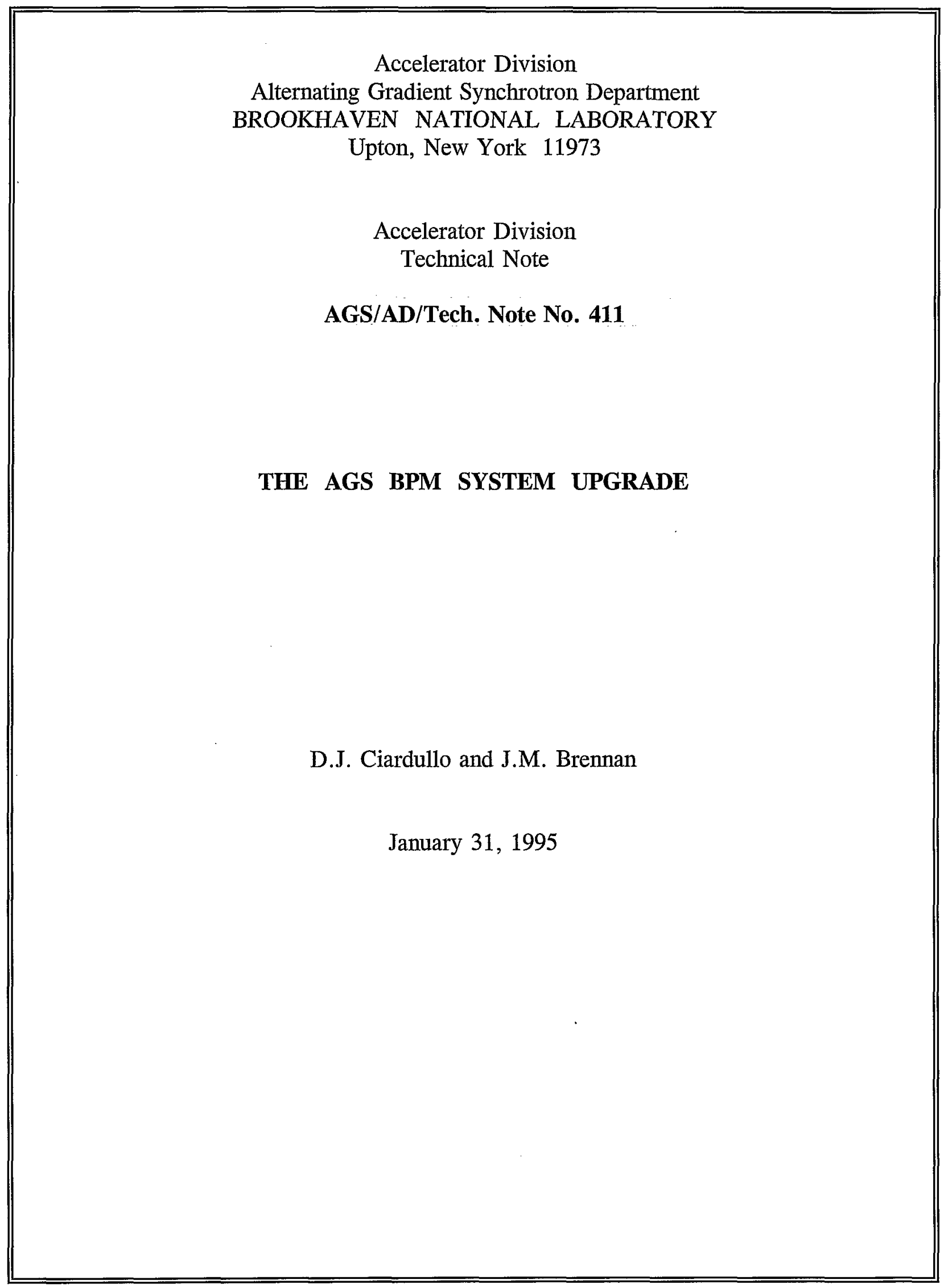




\section{The AGS BPM System Upgrade}

\section{Introduction:}

It is desired to upgrade the existing AGS BPM system to accommodate the wide range of intensities accelerated within the AGS, including protons, heavy ions and polarized protons. The current goal is to design an average orbit system capable of operating with beam intensities from $10^{9}$ to $10^{14}$ charges per pulse, using the presently installed AGS beam position detectors. To meet this challenge, new signal processing hardware needs to be designed. This document is a preliminary assessment of the basic objectives, philosophies and overall design concepts for a new average orbit system for the AGS. As such, feedback and discussion are encouraged.

\section{System Objectives:}

The following is a list of the key system objectives for this upgrade:

1. Build an average orbit measurement system using the existing, recently modified PUE detectors.

- The PUE detectors currently used in the AGS for orbit acquisition have been upgraded in recent years. Signal wiring internal to the detectors have been replace with radiation-hard shielded cable, and new vacuum flanges with BNC connectors have been installed. These detectors exhibit a linear differential response with beam position, and are considered to be of sufficient quality for use as the signal transducers for this upgrade.

2. Measure average orbits for intensities from $10^{9}$ to $10^{14}$ charges, over an $\mathrm{RF}$ range of $1.7 \mathrm{MHz}$ to 4.5 $\mathrm{MHz}$, with PPM compatibility.

- This 5 decade span of intensities implies a basic dynamic range requirement of $100 \mathrm{~dB}$; However, when the effects of non-zero beam position and bunch signal shape are taken into account, the dynamic range requirement for the peak voltage seen by a single PUE increases by an additional $20 \mathrm{~dB}$. [Note that beam positions several centimeters off-center can double the voltage seen on a particular electrode as compared with its "centered" value. In addition, the bunching factor for high intensity protons typically changes by a factor of five].

- The system is required to hold its position measurement accuracy stable over greater than an octave frequency sweep, concurrently with the intensity range requirement.

- PPM compatibility suggesets that the system should not require any ring access for amplifier "change-overs" between runs.

3. Build a reliable hardware system with a high degree of maintainability.

- This objective requires that there be no active electronics located inside the high radiation environment of the AGS ring, where high failure rates and lack of accessibility would dramatically increase system down time. 
4. Measure orbits accurately for a wide range of bunch lengths, from $10 \mathrm{nsec}$ to $300 \mathrm{nsec}$.

- Since the existing PUEs are of the electrostatic variety, their signals are a representation of the longitudinal charge density in the beam bunch. As such, the peak amplitude of the PUE signals will vary over a wide range, effectively increasing the dynamic range required of the system.

5. Measure average orbits with one or more bunches in the AGS (i.e., less than a "full" machine).

\section{Basic System Philosophies:}

In an attempt to realize the system objectives, design and development of the AGS BPM system upgrade will adhere to the following basic system philosophies:

- Keep active electronics out of the AGS ring!

Radiation levels are too high for reliable operation. Also, the need for access makes repairs and/or "on-the-fly" modifications extremely difficult (proven with the Booster BPM system).

- Build an Average Orbit System, not a universal PUE interface; This upgrade is not being designed to provide raw, wideband PUE signals. It will not provide trajectories (single turn orbits), and does not allow for bunch tracking. Due to the high dynamic range and broad bandwidth requirements of this upgrade, the purpose of the BPM system must be well defined in advance. The front end must essentially be a closed system in order to minimize corruption of the very low level PUE signals.

- Design in a well defined partition between the instrumentation and data acquisition portions of the system;

A well-defined division between these major sections increases the testability of each, as well as of the overall system. The processing electronics would provide as its output 216 channels of frequency-encoded position information (horizontal, vertical and "comfort" intensity). The Instrument Controller would then be responsible for inputing the FM information into the HP/Apollo system.

- Use frequency-domain signal processing techniques;

Position processing will be done in the frequency domain, operating on only one spectral component of the bunch signals from the AGS BPM detectors. With this method only a single component is needed, so there is no need to preserve the bunch shape fidelity of the PUE signals. This allows the use of heavy filtering techniques to boost their effective $\mathrm{S} / \mathrm{N}$ ratio.

Narrowband if processing also abolishes the need for fast, gated integrators; The implication here is that the bunch signals no longer need to be baseline restored. As such, the need for intricate, nanosecond timing is completely eliminated. In addition, the BPM measurement software does not have to compensate for the time dependant gain and offset errors normally associated with active integrators. 


\section{What the BPM Upgrade Would Include:}

The AIP provides funding for an upgrade of the hardware between the PUE transformers and the Instrument Controller (inclusive). In order to better describe the proposed upgrade, the BPM system will be divided into three basic areas:

1. Design of a new PUE signal delivery system;

2. Design of new average orbit processing electronics;

3. Design of the centralized peripheral hardware used to route the real-time orbit information from all BPM locations into one location (e.g., the Instrument Controller, multiplexer, etc.).

Figure 1 shows a preliminary "system concept" diagram, indicating the location of the different sections of the upgrade hardware. A brief description of what would be included in the upgrade follows.

\section{Design of a new PUE signal delivery system}

To satisfy the contradicting requirements of processing low intensity bunch signals while keeping only passive electronics in the ring, a systems approach must be taken when delivering the bunch signals to the electronics front end. Of paramount importance is the use of high quality (i.e., low loss, solid shield) coaxial cable; The PUE signals, as well as the electronics which process them, will be completely enveloped by an electrostatic enclosure (what can be thought of as a "faraday cage"). The plan is to use solid shield coaxial cable (which provides $100 \%$ rf shield coverage) to bring the PUE signals into the A-10, E-10 and $\mathrm{H}-10$ houses for analog processing. The processing hardware for each set of four BPM signals will utilize extruded aluminum-type enclosures in such a manner as to approximate, as closely as possible, one continuous extended electrostatic shield. This effectively prevents any if traveling up the shield of the coax from interfering with the processing electronics.

The upstream end of the signal delivery system includes new 16:1 wideband transformers. Since they are part of the PUE signal delivery system, the input and output side of these transformers are purposely not isolated. The function of these devices is to broadband match the impedance of each PUE to a resistive $50 \Omega$ (enabling the use of commercially available $50 \Omega$ cable), while minimizing the amount of signal loss due to capacitive electrode loading. At high frequencies, the transformer assemblies will terminate the 3nsec coaxial lead-out cables and suppress the quarter-wavelength resonance at $\approx 85 \mathrm{MHz}$. A preliminary design for an appropriate wideband autotransformer is currently being tested in the AGS.

\section{Development of new average orbit processing electronics}

It is planned to distribute the processing electronics equally among the A-10, E-10 and $\mathrm{H}-10$ houses. This satisfies the requirement of bringing the active components of the system out of the ring, while at the same time minimizing the lengths of the signal cables. For purposes of economy, cable runs from different detector locations will not be of matched length; However, the four PUE cables from each detector location need to be phase matched to one another.

The processing electronics located in the X-10 houses would provide localized gain and 
average orbit processing for 72 BPM detectors, each with dual-plane measurement capability. Current development efforts are directed toward designing a very high dynamic range rf receiverbased processing unit with continuous, automatic gain adjustment. In addition to an uninterrupted flow of average orbit information, this architecture also allows for a "comfort" output which indicates the beam intensity (This output is basically the AGC error voltage). Observation of this output gives an indication of whether or not the signal level is sufficient for successful operation of the system. All three analog outputs (horizontal position, vertical position and log intensity) are isolated using fiberoptic links with digital frequency modulation. Additional benefits of using this technique include a very high degree of noise immunity and protection against the amplitude variations normally associated with fiberoptic linearity and attenuation.

Localized test modules will be used for purposes of system test. This BITE (Built In Test Equipment) circuitry functions to verify proper operation of the remote processing electronics. $R F$ calibration and Local Oscillator (LO) signals are normally provided by the existing AGS LLRF system, and are shipped to the X-10 houses via analog fiberoptics. [A backup swept signal/LO pair would also reside in building 929, for the purpose of providing simple "go/no go" tests when the LLRF system is off-line]. Attenuation of the individual if test signals would occur at each set of processing electronics, so that variations in both intensity and position could be simulated. The BITE circuitry would provide:

1. Routing control of the switches which deliver the calibration signal to the processing electronics;

2. Swept-frequency rf calibration signals and LO (for use when the LLRF system is offline);

3. Common-mode amplitude control (in discrete steps) of the test signals to verify system dynamic range;

4. Differential-mode amplitude control of the test signals to simulate several different beam positions;

5. LO distribution, and distribution of the rf and LO test signals.

\section{Design of centralized peripheral hardware}

The peripheral hardware for the upgrade would reside in building 929 (possibly above the mezzanine). Plans include the design of a digital multiplexer to provide the MCR with $4 \mathrm{FM}$ signals of any combination of horizontal, vertical or intensity signals. Sending the FM signals to the MCR helps preserve signal integrety, but will require a piece of equipment at the receiving end to convert the modulated signals back to analog. The mux could also be used as a convenient "tie point" where the orbit information from any one or more BPM locations could be combined to obtain a true average orbit for the radial control loop. [This would decrease the sensitivity of the radial loop to loss of any one particular BPM location, but implies a position measurement bandwidth of $20 \mathrm{kHz}$. 
An Instrument Controller (also located in building 929) is required to sample and input to the HP/Apollo system the real-time orbit information from all BPM locations. Since the orbit information is always available, the sample times will be specified as time line events by the high level software. As such, the controller would include the hardware necessary to decode timeline events. The controller will receive frequency modulated orbit data directly, then use timed counters to evaluate the data; This eliminates the need to convert the information back to analog first, which tends to introduce additional errors into the data. Information from a total of 216 channels ( 72 locations $\times 3$ channels/location) would need to be gathered by the controller.

In addition to gathering data from the BPM system, the Instrument Controller will control the multiplexer described above. Hardware commands are also needed to set-up the BITE functions for system test. The following is a preliminary list of required controller commands/readbacks [all signals are active low]:

Command Name \# Bits Signal Type

/TEST_MODE 1 open collector

/SIM_POS 4 open collector

/SIM_POS_STAT 4 open collector

/SIM_INT $\quad 4 \quad$ open collector

/SIM_INT_STAT 4 open collector

/CAL

Mux bits

$1 \quad$ O.C., $1 \mu$ s pulse

32 open collector

Spare bits

Spare pulse
8 open collector

$4 \quad$ O.C., $1 \mu$ s pulse
Function

Puts electronics into testing mode.

Sets the diff mode gain of the test signals to simulate one of 16 discrete positions.

Status readback for SIM_POS command

Sets the common-mode gain of the test signals to simulate one of 16 discrete intensities.

Status readback for SIM_INT command

Informs system to output a zero-position test signal 5 milliseconds before T0 .

This is a first-pass guess based on an 8 bit word (to select one of 216 lines) for each of 4 lines to the MCR. May be refined further at a later time.

Spare bits for possible later use

Spare pulses for possible later use 


\section{AGS BPM UPGRADE}

OVERALL BLOCK DIAGRAM
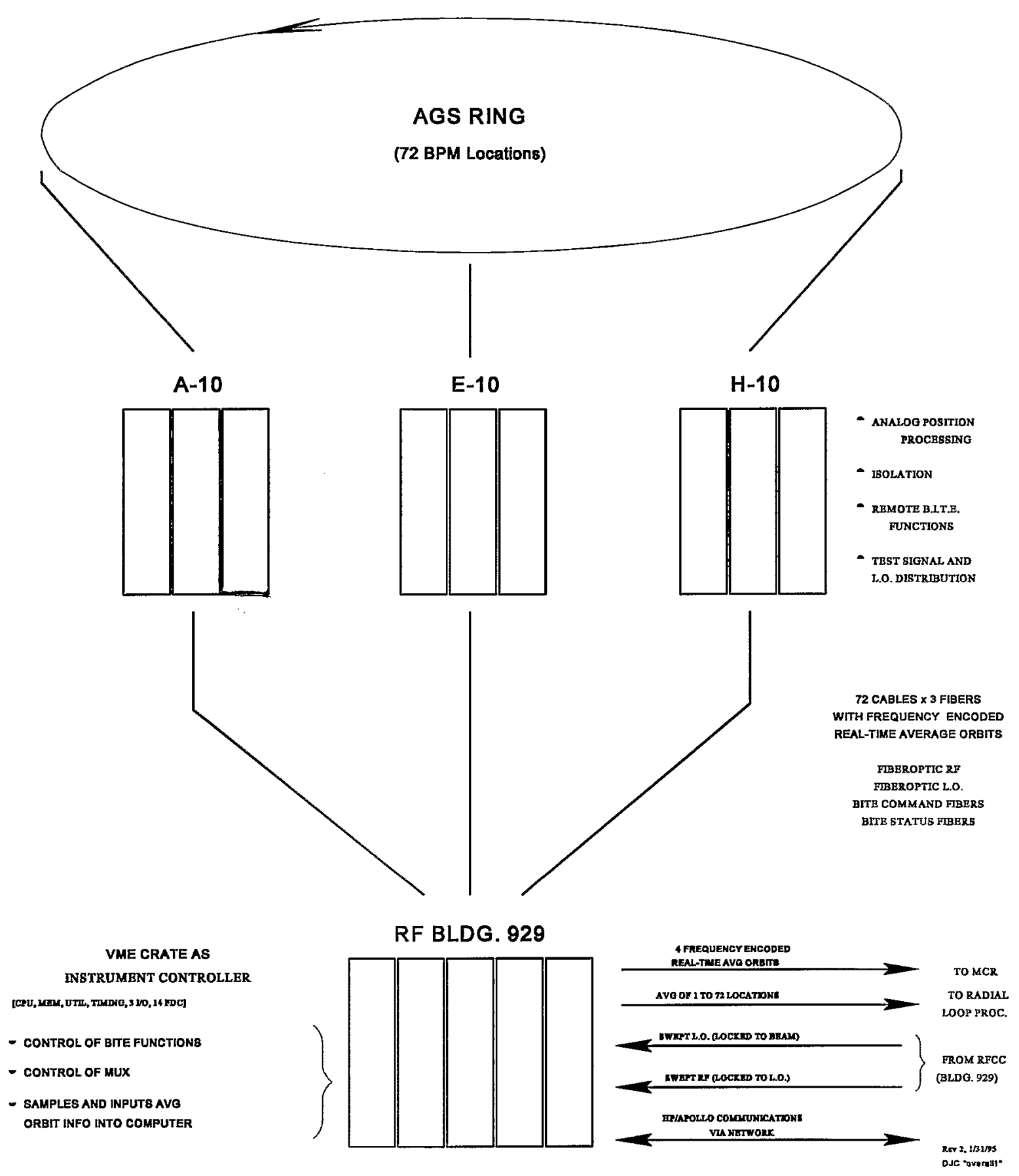

Figure 1 